\title{
La información financiera de calidad como facilitadora de gestión de riesgos y toma de decisiones
}

\section{Quality financial information as s facilitator of risk management and decision making}

\author{
MARISA MARChESANO \\ Académica, Universidad del Salvador y Universidad de Buenos Aires, Argentina. \\ Doctora en Contabilidad, Universidad de Buenos Aires. \\ Correo:marisamarchesano@gmail.com \\ Graciela María Scavone \\ Académica, Universidad del Salvador y Universidad de Buenos Aires, Argentina. \\ Doctora en Ciencias Económicas, Universidad de Buenos Aires.
}

Correo: gscavone@gmail.com

\section{RESUMEN}

Los cambios a los que se enfrentan las empresas son vertiginosos, y tanto la globalización como el avance tecnológico incrementan la volatilidad y el riesgo relacionados con los negocios. Ante esta situación, información de calidad sobre riesgos e incertidumbres se torna imprescindible. Es por ello que, en el presente trabajo, se dará cuenta de una investigación sobre la influencia de la incertidumbre en la calidad de la información financiera, con el objetivo de determinar mecanismos de medición y revelación que permitan acotarla, otorgando así un eficiente gerenciamiento del riesgo, a la vez que un adecuado proceso de toma de decisiones.

Se procedió en primer lugar a precisar los conceptos de riesgo e incertidumbre, así como a efectuar una descripción de los tipos de riesgo que un ente enfrenta, continuando con una conceptualización del gerenciamiento del riesgo y el proceso decisorio, que necesitan de información financiera de calidad para su desarrollo.

Se investigó sobre metodologías de medición que ponen su énfasis en la adecuada selección de las variables relevantes de un fenómeno, la determinación de la unidad de medida apropiada para la metrización y los instrumentos de medición a utilizar, además de considerar lo fenómenos desde un punto de vista sistémico. Se puse énfasis en la utilización de modelos matemáticos y en la información prospectiva. En cuanto a aspectos de revelación, la investigación se enfocó en las características de la información para ser considerada útil, así como en la claridad del discurso generado por el emisor de la información.

Palabras clave: riesgo, información financiera, medición, gestión de riesgo, toma de decisiones. 


\section{ABSTRACT}

The changes companies face is rapid, and both globalization and technological advancement increase volatility and business risk. In this situation, quality information on risks and uncertainties becomes essential.

That is why, in the present work, it will carry out an investigation of the influence of uncertainty on the quality of financial information, with the aim of determining measurement and disclosure mechanisms that make it possible to limit it, thus allowing efficient risk management, as well as an adequate decision-making process.

In the first place, the concepts of risk and uncertainty were specified, as well as a description of the types of risk that an entity faces, continuing with a conceptualization of risk management and the decision-making process that require quality financial information.

Research, was carried out on measurement methodologies that emphasize the appropriate selection of the relevant variables of a phenomenon, the determination of the appropriate unit of measurement for metrization and the measurement instruments to be used, in addition to considering phenomena from a point of systemic view. Emphasis was placed on the use of mathematical models and prospective information. Regarding disclosure aspects, the research focused on the characteristics of the information to be considered useful, as well as the clarity of the discourse generated by the issuer of the information.

Key words: risk, financial information, measurement, risk management, decision making.

\section{Introducción}

Las organizaciones o empresas enfrentan a diario situaciones de incertidumbre, las que, de no estar adecuadamente consideradas y reveladas, afectan la obtención de información contable de calidad, así como el proceso de toma de decisiones y gestión de riesgos por parte de los diferentes grupos de interés.

El riesgo está presente en todas las actividades de los agentes económicos. La vida de la empresa no es una excepción, y está sujeta a incertidumbre sobre una gran variedad de acontecimientos. Desde tiempos muy antiguos se reconoce la influencia de las fluctuaciones a diferentes factores de riesgo, como, por ejemplo: el clima o las variaciones de los precios de los insumos y productos finales, tasas de interés, tipos de cambio, entre otros. Es decir, se reconoce que, cualquier evento puede resultar mejor o peor de lo esperado, por lo que su impacto en una organización puede ser positivo o negativo.

El gerenciamiento del riesgo por parte de los emisores de los estados financieros se ha tornado imprescindible para la consecución de los objetivos de una organización y su sustentabilidad en el mediano y largo plazo. Su comunicación a los distintos grupos de interés, mediante información financiera de calidad es crucial para la toma de decisiones.

Según The Federation of European Risk Management Association, FERMA (2003), la gestión de riesgos es una parte esencial de la gestión estratégica de cualquier organización. Es un proceso por el que las empresas tratan los riesgos relacionados con sus actividades, a fin de obtener un beneficio sostenido de cada una de ellas, así como del conjunto de actividades.

La incertidumbre y el riesgo influencian la información suministrada por una organización, no obstante, existen formas que permiten acotar los efectos de los mismos, facilitando el proceso de gerenciamiento del riesgo y la toma de decisiones, tanto interna como de los grupos de interés relacionados con una organización. 


\section{1 objetivo}

El presente trabajo tiene como objetivo efectuar un análisis sobre la influencia de la incertidumbre en la información financiera, determinando mecanismos de medición y revelación que permitan acotarla, a fin de permitir un eficiente gerenciamiento del riesgo, a la vez que un adecuado proceso de toma de decisiones, tanto internas, como a nivel de los grupos de interés relacionados con la organización.

\section{2 aportes de la investigación}

Este trabajo tiene como objetivo efectuar un aporte doctrinario, mediante la profundización del conocimiento, difusión y propuestas de mejora, relacionadas con la problemática de medición y revelación en los estados financieros de aspectos relacionados con el impacto del riesgo y la incertidumbre.

\section{Revisión de la literatura}

\subsection{Conceptualización del riesgo y la incertidumbre}

Se hace necesario abordar ambos conceptos a fin de entender acabadamente las circunstancias bajo las cuales es necesario contar con información financiera de calidad, tanto para el gerenciamiento de un ente, como para la toma de decisiones de los grupos de interés relacionados con el mismo.

Los términos riesgo e incertidumbre suelen ser usados como sinónimos, sin embargo, hay una importante diferencia en el lenguaje económico entre ambos términos propuesta por Frank Knight (1921), que conviene clarificar y comprender. Se habla de incertidumbre cuando no es posible asignar una distribución de probabilidades al comportamiento futuro de una variable aleatoria, mientras que se habla de riesgo cuando si, se puede asignar una distribución de probabilidades a dicho comportamiento. Puede concluirse entonces, que el riesgo es solo una parte de la incertidumbre; aquella que podemos cuantificar y medir adecuadamente.

Según Prevé (2009), la distinción entre riesgo e incertidumbre ha sido objeto de encendidos debates entre los economistas. Por un lado, los economistas post-keynesianos argumentaban que esta es una distinción crucial para la comprensión de la economía y que la incertidumbre (no el riesgo) es la única fuente de aleatoriedad de la economía.

Para Viegas (2016, p.94), el riesgo es una contingencia o probabilidad de sufrir una pérdida o daño económico como resultado de la ocurrencia de un evento que altera las condiciones normales del desarrollo de la gestión. El desarrollo e implementación de un modelo de su control debe considerar distintos actores, entre ellos: los responsables de monitorear los riesgos y efectuar los controles (especialmente el órgano de gobierno, dentro de su alcance y de acuerdo con su responsabilidad en la gestión); la estructura a cargo del control y la administración de riesgos (pero también todas las unidades de la organización en sus diferentes niveles de responsabilidad).

La definición de riesgo tiene implícita la existencia de dos conceptos que son fundamentales para comprender el tema. Para poder tener una definición de riesgo es necesaria la existencia de un valor esperado, lo que conlleva la necesidad de que un hecho vaya a ocurrir en el futuro, si el hecho ocurriera en el presente no habría un valor esperado sino un valor cierto.

Por otro lado, es necesario que una variable se comporte de una manera que no se pueda predecir, en otras palabras, se necesitan variables cuyo comportamiento sea aleatorio. Esto es lógico dado que, si 
las variables tuvieran un comportamiento predecible con exactitud, el comportamiento esperado sería conocido y anticipado por los agentes económicos. Esto quiere decir que la existencia del riesgo depende de la existencia simultánea de dos factores: el futuro y las variables aleatorias.

\subsection{El riesgo y la vida empresaria}

En cuanto al riesgo y la vida empresaria, podría decirse que el riesgo es inherente a la empresa. Más específicamente, el riesgo justifica la existencia de las empresas, y legitima la existencia de beneficios corporativos. El beneficio generado por las empresas es una recompensa otorgada al inversor por la asunción de determinados riesgos. Si no hubiera riesgos, las empresas deberían generar una rentabilidad igual a la de un instrumento libre de riesgo y, en ese caso, todos los instrumentos financieros tendrían el mismo retorno: el retorno libre de riesgo.

Los inversores de una empresa enfrentan el riesgo total de la misma, que en principio estará relacionado con la volatilidad de los flujos de fondos y de los retornos respecto del valor esperado.

En cuanto al riesgo total enfrentado por los inversores, el mismo se puede dividir (LópezDumrauf, 2013) en:

$\checkmark$ "Riesgo único o diversificable

$\checkmark$ Riesgo sistemático, no diversificable o de mercado

El riesgo único es aquel que el inversor puede eliminar mediante la tenencia de un portafolio diversificado de acciones. Es el riesgo por el cual un mercado eficiente no paga ningún retorno, dado que la diversificación puede ser obtenida por el inversor sin costo alguno, simplemente mediante la inversión en diferentes acciones cuyas fluctuaciones de los retornos se compensan, al menos parcialmente, entre sí, logrando una disminución del riesgo total del portafolio.

El riesgo sistemático, no diversificable o de mercado es aquella porción del riesgo total del portafolio del inversor que no ha podido ser eliminado mediante la diversificación de la cartera. Este es el riesgo que miden los inversores profesionales y es el riesgo por el cual el mercado está dispuesto a pagar una rentabilidad adicional a quien esté dispuesto a asumirlo. Son ejemplos de tipos de riesgo no diversificable:

Riesgo de tipo de cambio

Riesgo de tasa de interés

Riesgo sobre el valor de los commodities

Riesgo de crédito, entre otros.

En la literatura financiera, se intenta modelizar de algún modo el comportamiento futuro de las variables aleatorias, pero al desconocer cómo se comportarán, solo pueden realizarse conjeturas que constituyen aproximaciones teóricas o modelos de comportamiento.

No obstante, estas conjeturas, basadas en los mejores elementos de juicio disponibles permiten llevar a cabo un adecuado gerenciamiento del riesgo.

Un riesgo tiene una causa y en caso de ocurrencia también implica un efecto y sus consecuencias. Es así como el desarrollo e implementación de un modelo para el control de estos factores (gestión de riesgo), se hace imprescindible, implicando el involucramiento de diversos actores, entre los cuales se destaca el órgano de gobierno de las empresas. 


\subsection{Definiendo gerenciamiento del riesgo}

La gestión de riesgo debe ser, sin duda, parte de la estrategia institucional y del proceso de toma de decisiones de un ente, a la vez que ser generadora de mejor información sobre los efectos de las decisiones tanto positivas como negativas (FERMA,2003)

Es un proceso continuo, efectuado por los administradores del ente, su dirección y el restante personal, en el cual se definen las estrategias de toda la empresa. Se diseña para identificar eventos potenciales que pueden afectar a la organización, gestionar riesgos dentro del nivel aceptado, proporcionando así una seguridad razonable sobre la consecución de los objetivos de la entidad (Committee of Sponsoring Organizations of the Treadway Commission-COSO, 2013)

Estas capacidades, sin duda, ayudan a la dirección a alcanzar los objetivos de rendimiento y rentabilidad propuestos, previniendo la pérdida de recursos. Permite, asimismo asegurar información efectiva, el cumplimiento de las leyes y regulaciones y evitar inclusive daños en la reputación del ente.

No existen dudas que las organizaciones seguirán enfrentando un futuro lleno de volatilidad, complejidad y ambigüedad. La gestión de riesgo empresarial constituye así, una parte importante de cómo una organización se maneja y prospera a través del tiempo.

Con independencia del tipo o tamaño de las organizaciones, las estrategias deben permanecer fieles a su misión. Todas las entidades necesitan exhibir rasgos que conduzcan a una respuesta efectiva al cambio, incluyendo un ágil proceso de toma de decisiones, adaptación al cambio y reposicionamiento, manteniendo el nivel de confianza entre las partes interesadas.

La cobertura de riesgos no disminuye ni anula las fluctuaciones del factor riesgo, sino que es un proceso por el cual una empresa transfiere aquellos riesgos a terceros que, habitualmente cobran un precio por hacerlo.

La correcta implementación de procesos con enfoque de administración de riesgos hace que deban definirse el ámbito y las políticas de seguridad de la información. Debe analizarse el proceso de captura de la información para la valoración de los riesgos y evaluar los procesos de registro con la finalidad de asegurar la fiabilidad de la base de datos. Por lo tanto, de lo antedicho se desprende la necesidad de información financiera de calidad para un adecuado proceso de gestión de riesgo.

\subsection{El proceso decisorio}

“Una situación de decisión, para la teoría de la decisión, es el conjunto de los siguientes elementos definidos y evaluados por un decididor-persona determinando: objetivos, cursos de acción alternativos, estados aleatorios, probabilidades y resultados" (Pavesi, 1994, p.212).

En relación con el proceso decisorio, Brim (1962, p.9), propone los siguientes pasos para llevar a cabo el proceso decisorio a saber: “a) identificación del problema, b) obtención de la información necesaria, c) la producción de soluciones posibles, d) evaluación del tipo de soluciones posibles, e) la selección de una estrategia de desempeño, y f) el desempeño de la o las acciones seleccionadas."

Por otra parte, Fowler Newton (2007), expresa que el mismo implica la realización continua de las siguientes actividades: 
- Detectar disparadores del proceso de decisión

- Identificar los cursos de acción posibles

- Estimar los posibles efectos de esos cursos de acción

- Seleccionar un curso de acción

- Actuar en concordancia con el curso de acción seleccionado

- Considerar los resultados de lo actuado

- Comparar los resultados reales con los esperados, estableciendo y analizando las causas de los desvíos.

Ante la necesidad de obtener información necesaria para satisfacer el proceso decisorio surge el sistema de generación de información financiera como recurso esencial para dicho proceso. El mismo debe ser diseñado de forma tal que genere información de calidad para llevar a cabo el proceso analizado.

\subsection{Metodologías de medición en entornos de riesgo e incertidumbre}

La medición supone un proceso de representación de un fenómeno empírico en el mundo de las matemáticas. Esta representación pocas veces llega a ser perfecta, especialmente en lo que se refiere a disciplinas sociales.

Sabido es que la utilidad de la información financiera mejora a partir de la fundamentación teórica de la medición y emisión de información. La actividad académica de investigación colabora con el tratamiento riguroso de ciertos elementos del discurso contable.

Se ha puesto de manifiesto el escaso grado de conocimiento de las características del proceso de medición en contabilidad, lo que impide la comprensión adecuada de la información producida por los diferentes sistemas contables.

Mattessich (2002), ha mostrado preocupación por las condiciones de la medición al definirlas como la asignación de numerales a objetos o eventos siguiendo ciertas reglas.

Los problemas relacionados con los aspectos de medición contable se relacionan sustancialmente con la adecuada selección de las variables relevantes que caracterizan los fenómenos tratados, la definición adecuada de la unidad de medida y la elaboración de instrumentos de medición que permitan captar de forma apropiada las propiedades del fenómeno analizado.

La contabilidad debe abordar realidades inciertas y subjetivas frente a las cuales se ven inmersas tanto las empresas como la sociedad, buscando el reflejo más fiel y consciente de los fenómenos organizacionales y sociales de los que se ocupa.

Para el reflejo de tales fenómenos y otros con características predictivas de caos o turbulencia, debe recurrir, sobre todo en lo que hace al proceso de medición a otras ciencias que presenten su mayor herramienta en los potenciales desarrollos matemáticos.

Es así como recurriendo a los modelos matemáticos desarrollados por Black, Scholes y Merton (1973); Cox, Ross y Rubinstein (1979), se puede determinar el valor de uno de los instrumentos financieros derivados como las opciones.

Sánchez-Villamil (2015), al conceptualizar lo que denomina ciencias de la complejidad se refiere a la necesidad por parte de la contabilidad, de abordar realidades inciertas y subjetivas. Estas ciencias presentan su mayor 
herramental en los desarrollos matemáticos, y han tomado cuerpo gracias al desarrollo de sistemas computarizados. Lo que caracteriza la complejidad, según Sánchez-Villamil, es el quiebre de las comprensiones disciplinares y monolíticas del conocimiento, a fin de abarcar enfoques transversales e interdisciplinarios.

Para Barbei et al (2016), el grado en que las mediciones son consistentes dependerá de la variabilidad del objeto, del contexto, de los instrumentos de medición y del observador.

Hendriksen (1970) considera que una estructura teórica contable debería contener los siguientes elementos:

- La evaluación de los posibles procesos de medición y descripción a fin de comunicar la información sobre la entidad y su medio.

- La evaluación de las limitaciones a la medición y la descripción de la entidad y su medio.

- La denominación de lo que el autor define como una estructura y un modelo para la recopilación y elaboración de datos y para resumir y dar cuenta de la información pertinente.

López-Díaz, (2012, p. 19) establece una serie de requisitos a ser complementados antes del inicio del proceso de medición:

- Definir las dimensiones relevantes del fenómeno en el mundo real que se mide;

- Exponer las escalas de medición establecidas, en el sentido de que estén definidas las relaciones entre los números que servirán para realizar las comparaciones entre objetos;

- Definir las unidades empleadas, según cual sea la característica o dimensión del fenómeno real que pretende captarse, $\mathrm{y}$
- definir la operación de la que se obtiene un número asignada a cada objeto referido a una escala de medición.

Por otra parte, Viegas \& Pérez (2011), consideran que, si bien los actores que operan en los mercados reconocen la importancia de contar con información financiera, la misma no sería suficiente para reducir los riesgos, ya que no se obliga a las diferentes entidades a que expongan en forma sistemática su situación acerca del futuro y su evolución dentro del contexto en que actúa. Los autores proponen la generación de estados financieros prospectivos, a imagen y semejanza que los de cierre de ejercicio, generados en función a hipótesis y supuestos hipotéticos, tanto para un adecuado gerenciamiento del riesgo, como para brindar a los diferentes grupos de interés vinculados con el ente, un proceso de toma de decisiones que contenga elementos de las proyecciones de la gerencia del ente en cuestión.

\subsection{Aspectos de revelación a tener en cuenta para acotar la incertidumbre}

Ya en el contexto de revelaciones sobre riesgos, es decir, en cuanto a la transmisión de información a terceros que constituyen grupos de interés relacionados con el ente, lo importante es la forma en que el emisor de estos informes transmite las distintas formas de riesgo, a fin de que sean acabadamente comprendidas por los usuarios de los estados financieros.

Para Scavone (2002), uno de los principales objetivos de la información financiera consiste en la transmisión del mensaje que surge de la misma entre los emisores y los usuarios de dicha información. Dicha comunicación se lleva a cabo mediante la utilización de palabras técnicas y específicas, características de un lenguaje particular. La correcta interpretación de 
los mensajes por parte de los grupos de interés, tiene como requisito la emisión de mensajes claros, legibles y comprensibles. A su vez, el emisor del mensaje es quien modela la reacción del receptor, es decir que existen entonces dos partes: el constructor y el decodificador del mensaje. Asimismo, agrega, que el mensaje que se comunica a través de la información financiera podría ser utilizado para generar en los usuarios sentimientos diferentes a la verdad. También recomienda la utilización de un lenguaje claro, otorgando debido cuidado a la gramática empleada, lo que incluye un análisis de la complejidad de las estructuras sintácticas, la longitud de las oraciones y la adecuada selección de las palabras.

El Marco Conceptual de las Normas Internacionales de Información Financiera (NIIF), emitido por el International Accounting Standards Board, IASB (2018), al hacer referencia a las características cualitativas que debe reunir la información financiera para considerarse útil, las subdivide en características fundamentales y de mejora. Señala así, que constituyen parte de las primeras la relevancia y la representación fiel, en tanto que identifica como características de mejora las siguiente: comparabilidad, verificabilidad, oportunidad y comprensibilidad.

La suficiencia de los datos de los informes es vital para hacerlos comprensibles. Su origen debe hallarse en una base de datos sistematizada, así como su estructura permitir análisis y comparaciones. Este objetivo es solo alcanzable si se posee un sistema que provea información completa, confiable y oportuna, que permita monitorear la exposición al riesgo, siendo a la vez útil para la toma de decisiones.

Resulta conveniente evitar generalizaciones, fechar informes, proveer un marco de re- ferencia, a fin de que el usuario de la información tenga bases para efectuar determinadas comparaciones consideradas útiles.

Debería evitarse la abundancia de información de tipo narrativo, a fin de que no se vean alteradas la sistematicidad, comparabilidad e integridad de los datos, facilitando la distinción de la estructura organizativa de la información.

"La calidad de los datos no debe subestimarse: ninguna herramienta puede compensar la mala calidad de los datos. La información de mala calidad afecta los resultados y puede tener serias consecuencias de interpretación que afecten los procesos de toma de decisiones" (Viegas, 2016, p. 96).

Por otra parte, el mismo autor, en su tesis doctoral, aborda la problemática relacionada con la información en el marco de la gestión de riesgo. Allí explicita que los sistemas de información deberían utilizar indicadores basados en estándares que permitan medir adecuadamente los sucesos pasados, presentes y futuros.

La demanda de información sobre riesgos de calidad puede ser vista como una tendencia más amplia de insatisfacción con las limitaciones de la información financiera histórica, además de la necesidad de información prospectiva.

\section{Metodología}

Se llevó a cabo un relevamiento de metodologías de medición y revelación, que se consideraron relevantes, a través del análisis de bibliografía tanto internacional como local en relación a la temática desarrollada, con la finalidad de identificar de aquellas propuestas que permitan reducir la incertidumbre, recurriendo a fuentes primarias como libros, publicaciones periódicas de reconocimiento 
internacional, tesis de doctorado, trabajos de investigación y a la experiencia de distintas organizaciones.

Además, se analizaron encuestas desarrolladas por organismos internacionales de máximo nivel, sobre los requerimientos de los usuarios externos en relación a la información sobre riesgos e incertidumbre de un ente y sus políticas de gerenciamiento: Association of Chartered Certified Accountants (2014), the Institute of Chartered Accountants in England and Wales (2011); Cabedo Semper, Tirado Beltran (2015) y los economistas Zhang, Zhang, Seier (2015).

\section{Análisis de resultados}

A fin de brindar un panorama sobre la situación a nivel internacional y su evolución en cuanto a información sobre riesgos e incertidumbres, se procederá a un análisis e interpretación de los resultados de las encuestas de opinión llevadas a cabo por the Association of Chartered Accountants (2014), the Institute of Chartered Accountants in England and Wales (2011), Cabedo-Semper y Tirado-Beltran (2015) y Zhang, Zhang, Seier (2015). En ellas se reflejan sustancialmente, los puntos de vista de los usuarios en relación a la información sobre incertidumbres y riesgos que se les suministra.

Entre las consideraciones a las que se arribaron, se destacan las siguientes:

- Las empresas parecen preferir los formatos de divulgación relativamente complicados, pero más discretos, que los formatos simples, pero más reveladores.

- La información sobre riesgos debería ser principalmente cuantitativa y debe actualizarse en forma continua.

- Se solicita la localización de la información sobre riesgos en una sección específica de la información que emiten las organizaciones, la que actualmente se encuentra dispersa en diferentes documentos y secciones dentro de los mismos.

- Se reclama que la misma sea revisada por un auditor independiente. Aquí las autoras sugieren recurrir a metodologías de revisión como los encargos de Aseguramiento.

- Se plantea que actualmente la información bajo análisis es sustancialmente cualitativa y genérica.

- Se solicita que se informe para cada uno de los principales riesgos, la valoración de los posibles impactos sobre la empresa.

- Perciben la información como dudosa, dado que presentan un carácter repetitivo, genérico $y$, con ciertas salvedades, similar entre empresas.

El contexto actual con un elevado nivel de incertidumbre ha motivado una creciente demanda de información en los estados financieros encaminada a que los usuarios externos tengan un mayor conocimiento de la realidad empresarial y valoren entonces los riesgos e incertidumbres a que se encuentran expuestas sus actividades.

Del relevamiento empírico llevado a cabo surge que la información de riesgos que actualmente ofrecen las empresas es cualitativa y genérica, cuando en realidad se hace necesario que la información divulgada sea principalmente cuantitativa.

\section{Conclusiones}

Como resultado de la investigación, se ha concluido que, a fin de obtener información de calidad que permita una adecuada gestión de riesgos y toma de decisiones, se considera procedente considerar los aspectos que se detallan a continuación: 
Partiendo de la base que de cualquier medición supone un constructo hipotético sujeto a contrastación, se propone un proceso de medición que contemple los siguientes aspectos:

- Elección del fenómeno a describir, el cual debe formar parte del dominio contable;

- Planteo del marco teórico conceptual en el cual se pueda establecer el significado que toma para cada concepto dentro del modelo de medición en particular;

- La determinación de los fines perseguidos por el observador del proceso;

- La selección de las variables relevantes para la descripción del fenómeno y las dimensiones que se pretenden operacionalizar de las mismas;

- La selección de la unidad de medida más apropiada, es decir, la metrización del ámbito en particular;

- La determinación de los instrumentos de medición a utilizar;

- La consideración de los fenómenos que se están presentando desde un punto de vista sistémico, es decir afectado por un conjunto de interacciones con otros fenómenos.

Resulta de suma importancia recurrir a otras ciencias que presentan su mayor herramienta en potenciales desarrollos matemáticos, tal el caso de los desarrollos de Black yScholes, 1973; Cox, Ross y Rubinstein, 1979.

En cuanto a aspectos de revelación, se detallan aspectos a considerar para la emisión de información financiera de calidad:

- Considerar las siguientes cualidades que deber revestir la información: relevancia, imagen fiel, comparabilidad, comprensibilidad, oportunidad y verificabilidad.
- Utilización de un lenguaje claro, prestando especial atención a la gramática del discurso.

- Evitar generalizaciones, fechar informes, proveer un marco de referencia, a fin de posibilitar comparaciones por parte de los usuarios.

- Evitar la abundancia de información de tipo narrativo, a fin de no afectar la sistematicidad, comparabilidad e integridad de la información.

- No utilizar el lenguaje contable como modelador del comportamiento del usuario para transmitir sucesos o situaciones irreales.

- Los datos de los informes, en su origen, debe hallarse en una base de datos sistematizada, y su estructura debe permitir análisis y comparaciones. Para el logro de este objetivo un sistema debe proveer información completa, confiable y oportuna, que permita monitorear la exposición al riesgo.

- Los datos de entrada deben ser de calidad y los procesos de generación de información, seguros.

Los informes financieros de calidad aumentan la confianza de los inversores, no solo en términos de los riesgos que se discuten, sino porque permiten revisar la gestión que de los mismos lleva a cabo la gerencia de una empresa.

Se hacen necesarios modelos de información basados en hechos y sucesos prospectivos, bajo la forma de estados financieros prospectivos emitidos en forma sistemática, conjuntamente con el estado de cierre de ejercicio, a la vez balances de responsabilidad social empresaria, a fin de poder encarar exitosamente los nuevos y cambiantes desafíos que se presentan. 
Queda abierta la posibilidad de investigaciones futuras, avanzando e innovando en metodologías de medición y revelación en contextos de incertidumbre.

\section{Referencias}

Association of Chartered Certified Accountants (ACCA), (2014), Reporting Risk. http:/ / www.accaglobal.com/content/dam/ acca/global/PDF-technical/ financial-reporting/pol-afb-rr.pdf

Barbei, A; NeIrA, G., (2016), Contabilidad Financiera: Modelos decisorios, necesidades de los usuarios y emisión de información. Centro de Estudios en Contabilidad Internacional. Facultad de Ciencias Económicas. Universidad de La Plata. http:/ / www.econo.unlp.edu.ar/ uploads/docs/cecin_documento_de_trabajo_nro_26.pdf

Black, F., Scholes, M. (1973), The Pricing of Options and Corporate Liabilities, The Journal of Political Economy, 81(3) 637-654. Disponible en: http://www.jstor.org/stable/1831029.

Blejer, D., Thomas, M. (2017), NIIF 9 y contabilidad alineada con prácticas de administración de riesgo. KPMG. https://home.kpmg.com/ar/ es/home/Tendencias/2017/10/niif-9-y-contabilidad-alineada-con-practicas-de-administracion-de-riesgos.html

BRIM, O. (1962), Personality and decision processes. Studies in the social psychology of thinking. (Vol.2). Standfor Univerity Press.

Cabedo-Semper, J., Tirado-Beltrán, J. (2015), La información de riesgos en los Estados Financieros: situación actual y perspectivas futuras. (Revista 110, XXI Premio AECA de artículos en Contabilidad y Administración). Asociación Española de Contabilidad y Administración de Empresas. http://www. aeca1.org/revistaeca/revista110/110.pdf
Committee of Sponsoring Organizations of the Treadway Commission, COSO, (2017), Enterprise Risk Management. Integrated with Strategy and Performance. Executive Summary. https://www.coso.org/Documents / 2017-COSO-ERM-Integrating-with-Strategy-and-Performance-Executive-Summary.pdf

Committee of Sponsoring Organizations of the Treadway Commission, COSO, (2013), Internal Control - Integrated Framework. Committee of internal Control.

Committee of Sponsoring Organizations of the Treadway Commission. COSO -ERM (2017) Guidance for applying Enterprise Risk Management (ERM) to Environmental, Social and Governance (ESG)-related risks.

Cox, J., Ross, S., Rubinstein, M., (1979), Options princing: a simplified aprroach. Journal of Financial Economics $N^{\circ}$ 7, págs. 229 a 263.

Di Ranni, M., (2016), El Sistema Contable Integrado y la Eficiencia de las Funciones de Gestión. (Tesis de Doctorado). Facultad de Ciencias Económicas. Universidad de Buenos Aires. http: / / bibliotecadigital.econ.uba.ar / ?c=tesis\& $\mathrm{a}=\mathrm{d} \& \mathrm{cl}=\mathrm{CL} 1$

Federation of European Risk Management Association (2003), Estándares de Gerencia de Riesgo. Reino Unido, Airmic, Alarm, Irm, (2002), traslation copyright FERMA (2003).

Fowler Newton, E. (2007), Análisis de Estados Contables. 3ra. Edición. La Ley.

Hendriksen, E. (1970), Accounting theory. Irwin Professional Publishing

Institute of Chartered Accountants in England ANd Wales (ICAEW), (2011), Reporting Business Risks: Meeting Expectations. Information for better markets initiative. Reino Unido de la Gran Bretaña. ISBN 978-0-85760-291-6. https: / / www.icaew.com/-/media/corporate/files/technical/financial-reporting/in- 
formation-for-better-markets/ifbm/rbr-final. ashx

INTERNATIONAL ACCOUNTING STANDARDS BOARD (2018), Conceptual Framework of Financial Reporting. https:/ /www.iasplus.com/en/ standards/other/framework

Knight, F. (1921) Risk, Uncertainty and Profit. New York, USA. Cambridge University Press. https:/ / fraser.stlouisfed.org/files/docs/publications/books/risk/riskuncertaintyprofit. pdf

López DíAz, A. (2012), La medición y valoración en contabilidad. Análisis conceptual. Facultad de Ciencias Económicas y Empresariales de la Universidad Complutense.

López-Dumrauf, G. (2013), Finanzas Corporativas. Un enfoque latinoamericano. Ed. Alfaomega.

Mattessich, R. (2002), Contabilidad y Métodos Analíticos: medición y proyección del Ingreso y la Riqueza en la Microeconomía y la Macroeconomía. Traducción García Casella, C, Rodriguez, M. La Ley.

Pavesi, P. (1994), Lo normativo y lo descriptivo y su conflicto con la paraxiologías: el caso de la teoría de la utilidad (de predicadores y pecador). [Tesis Doctoral, Universidad de Buenos Aires]. http:/ / bibliotecadigital.econ.uba.ar/econ/ collection/tesis/browse/CL2/16

Prevé, L. (2009), Gestión del Riesgo. Un enfoque estratégico. Ed. Temas.
SÁNCHEZ-VIllamil, G. (2015), La incertidumbre en la contabilidad desde las ciencias de la complejidad. Facultad de Contaduría Pública. Universidad de la Gran Colombia. https:/ / www. ugc.edu.co/documentos/contaduria/publicaciones/Revista_Hilando_Cuentas_UGC. pdf

Scavone, G. (2002), Comportamiento de las variables que influyen en la percepción y comprensión de la información contable. [Tesis Doctoral, Universidad de Buenos Aires]. http:// bibliotecadigital.econ.uba.ar/download/tesis/1501-1164_ScavoneGM.pdf

Túa Pereda, J. (1995), Lecturas de Teoría e Investigación Contable. Centro Interamericano Jurídico Financiero.

Viegas, J. (2016), Marco teórico-conceptual de las planificaciones operativas y estratégicas. Un modelo contable prospectivo y de sustentabilidad. [Tesis Doctoral, Universidad de Buenos Aires]. http://bibliotecadigital.econ.uba.ar/ econ/collection/tesis/browse/CL2

Viegas, J., Pérez, O. (2011), Confección de Estados Financieros Prospectivos y Control de Auditoría. Osmar Buyati

ZHANG, Y., ZHANG, H., SEIER, M. (2015), Impact of Information Disclosure on Prices, Volume and Market Volatility: An Experimental Approach. The Journal of Behavioral Finance: Volume 16, $\mathrm{N}^{\circ}$ 1. http:/ / dremanbehavioralfinance.org/journals/abstracts/Volume\%20 16.html 